\title{
Surface Wind Regionalization over Complex Terrain: Evaluation and Analysis of a High-Resolution WRF Simulation
}

\author{
Pedro A. Jiménez,*,+ J. Fidel GonzÁlez-Rouco,* Elena García-Bustamante,*,+

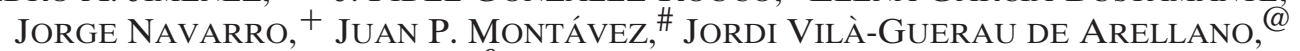 \\ JiMY DUDHIA, \& AND ANTONIO MUÑOZ-ROLDAN** \\ * Departamento de Astrofísica y Ciencias de la Atmósfera, UCM, Madrid, Spain \\ ${ }^{+}$División de Energías Renovables, CIEMAT, Madrid, Spain \\ \# Departamento de Física, UM, Murcia, Spain \\ @ Meteorology and Air Quality Group, Wageningen University, Wageningen, Netherlands \\ $\&$ Mesoscale and Microscale Meteorological Division, National Center for \\ Atmospheric Research, ${ }^{+}{ }^{+}$Boulder, Colorado \\ ** CIEMAT, Madrid, Spain
}

(Manuscript received 16 December 2008, in final form 8 September 2009)

\begin{abstract}
This study analyzes the daily-mean surface wind variability over an area characterized by complex topography through comparing observations and a $2-\mathrm{km}$-spatial-resolution simulation performed with the Weather Research and Forecasting (WRF) model for the period 1992-2005. The evaluation focuses on the performance of the simulation to reproduce the wind variability within subregions identified from observations over the 1999-2002 period in a previous study. By comparing with wind observations, the model results show the ability of the WRF dynamical downscaling over a region of complex terrain. The higher spatiotemporal resolution of the WRF simulation is used to evaluate the extent to which the length of the observational period and the limited spatial coverage of observations condition one's understanding of the wind variability over the area. The subregions identified with the simulation during the 1992-2005 period are similar to those identified with observations (1999-2002). In addition, the reduced number of stations reasonably represents the spatial wind variability over the area. However, the analysis of the full spatial dimension simulated by the model suggests that observational coverage could be improved in some subregions. The approach adopted here can have a direct application to the design of observational networks.
\end{abstract}

\section{Introduction}

The study of wind field over a specific region can be undertaken from a purely observational standpoint (e.g., Baker et al. 1978; Martner and Marwitz 1982; Wendland 1982; Klink 2002; Jiménez et al. 2009). Alternatively, this can be taken on through model-based approaches that allow for a more complete understanding of the physical processes and mechanisms involved (e.g., Mahrer and Pielke 1977; Mahrer et al. 1985; Rife et al. 2004).

\footnotetext{
++ The National Center for Atmospheric Research is sponsored by the National Science Foundation.
}

Corresponding author address: J. Fidel González-Rouco, UCM, Facultad de Ciencias Físicas, Avd. Complutense s/n, 28040 Madrid, Spain.

E-mail: fidelgr@fis.ucm.es
Observational-based studies target an accurate representation of wind variability at the regional level, assuming that local in situ information is representative for some area around the site and that the integration of this information for all sites is sufficient to provide good regional-scale coverage. However, the reliability on the representation of the regional flow can be hampered by the quality and availability of wind records. Topography also impinges on the regional representation by increasing the complexity of the flow as a consequence of its interaction with large-scale dynamics and radiation (Whiteman 2000).

Model-based assessments lean on models of either diagnostic or prognostic type. The diagnostic models blend observational information and modeling to provide a physically consistent interpolation of the wind field. This is achieved by imposing constraints such as the conservation of mass through the continuity equation (e.g., 
Sherman 1978; Goodin et al. 1980; Ludwig et al. 1991; Endlich et al. 1982). Somewhat more elaborate diagnostic models are based on the theory of Jackson and Hunt (1975) and its extension to three dimensions by Mason and Sykes (1979), which linearized the equations of motion to obtain an analytical solution (e.g., Walmsley et al. 1982; Troen and Petersen 1989). These models provide satisfactory results over hilly terrain (Jenkins et al. 1981; Mason and King 1984), but their application to steep slopes typical of complex terrain regions can be problematic because of the linearization applied to the governing equations. In addition, diagnostic models do not take into account thermal effects. Thus, their use is designed to evaluate the effects of orography on steady mean wind flows (Ratto et al. 1994).

A more realistic representation including thermal effects is provided by prognostic mesoscale models (Pielke 2002). These models numerically solve the Euler equations to derive physically consistent meteorological fields, usually after applying some simplifications such as Reynolds averaging (Reynolds 1895) and representing subscale physical processes in the form of parameterizations (e.g., Black 1994; Grell et al. 1994; Cotton et al. 2003; Skamarock et al. 2005). The initial and boundary conditions necessary to perform the simulation are obtained from a general circulation model. The accuracy of the mesoscale model in representing the wind field can be evaluated by comparing the simulations with independent observational information (e.g., Mahrer et al. 1985; Rife et al. 2004; Zagar et al. 2006). Additionally, hybrid approaches can enhance the spatial resolution of a mesoscale simulation using a diagnostic model (e.g., Ludwig et al. 2006); however, their reliability is conditioned to that of the mesoscale simulation and the suitable representation of physical processes.

The evaluation of numerical simulations has some uncertainties when comparing with local information is involved (von Storch 1995). Some works compare in situ observations with the nearest simulated grid points (Cox et al. 1998; Hanna and Yang 2001; Buckley et al. 2004). However, two main reasons are worth stressing as responsible for the uncertainty introduced in this particular type of comparison. First, the simulated variables represent averaged quantities over volumes with homogeneous properties because of the spatial discretization and the Reynolds averaging. Therefore, its comparison with in situ observations is controversial, mostly at those locations that are considerably affected by local features. Second, the spatial discretization smoothes the complexity of surface physical properties such as orography. This can potentially lead to a situation in which the simulated volume that includes the actual location of the observational site may not be the most suitable one to represent the observational variability; instead, nearby volumes may be more appropriate. This last problem is referred to as the "representativeness error." There are different methodologies to mitigate such adverse effects. For instance, the enhanced resolution provided by hybrid approaches makes point comparisons more reasonable; alternatively, the filtered spatial patterns of the leading EOFs calculated with observations and simulations can be compared (e.g., Volmer et al. 1984). An interesting approach was proposed by Reid and Turner (2001) that compared the volume-averaged quantities simulated at a coarse horizontal resolution $(40 \mathrm{~km})$ against averaged observations within subjectively defined subregions. They showed that averaging observational series to obtain regional ones filters out local variability (noise), thus enhancing the regional signal. This led to stronger relationships between model and data than in the traditional nearest gridpoint comparison. Reid and Turner (2001) highlight the advantages of an evaluation performed at the regional scale, although their definition of subregions is subjective.

Our work uses a simulation performed with the Weather Research and Forecasting (WRF) mesoscale model (Skamarock et al. 2005) and the Reid and Turner (2001) concept of regional evaluation to analyze the daily wind variability over a complex terrain region. A more objective evaluation is attained by using an automated approach to identify the subregions without any a priori knowledge of the wind behavior over the area of study. The Comunidad Foral de Navarra (CFN) in the northeast of the Iberian Peninsula (Fig. 1) was selected for this case study. The CFN presents a reasonably dense surface meteorological network that has made it possible to undertake various analyses of the properties of the wind field within this region (García et al. 1998; Torres et al. 1999, 2005; García-Bustamante et al. 2008, 2009; Jiménez et al. 2008, hereinafter JEA08; 2009). In particular, JEA08 studied a subset of the available observational data to group together the sites with similar temporal wind variability.

The present study extends and completes JEA08 and uses their methodologies and classification of observational regions to assess the reliability of the WRF numerical simulation in reproducing them. The whole available observational period from 1992 to 2005 is simulated with a spatial resolution of $2 \mathrm{~km}$. This provides a long simulation with both a high spatial and temporal resolution, comparable to that of the observational dataset. The innovative character of the WRF simulation relies on its length and spatial resolution (over $13 \mathrm{yr}$ of simulation at $2 \mathrm{~km}$ of horizontal resolution). The value of the WRF simulation should be viewed in the context of the computational costs of running prognostic mesoscale 


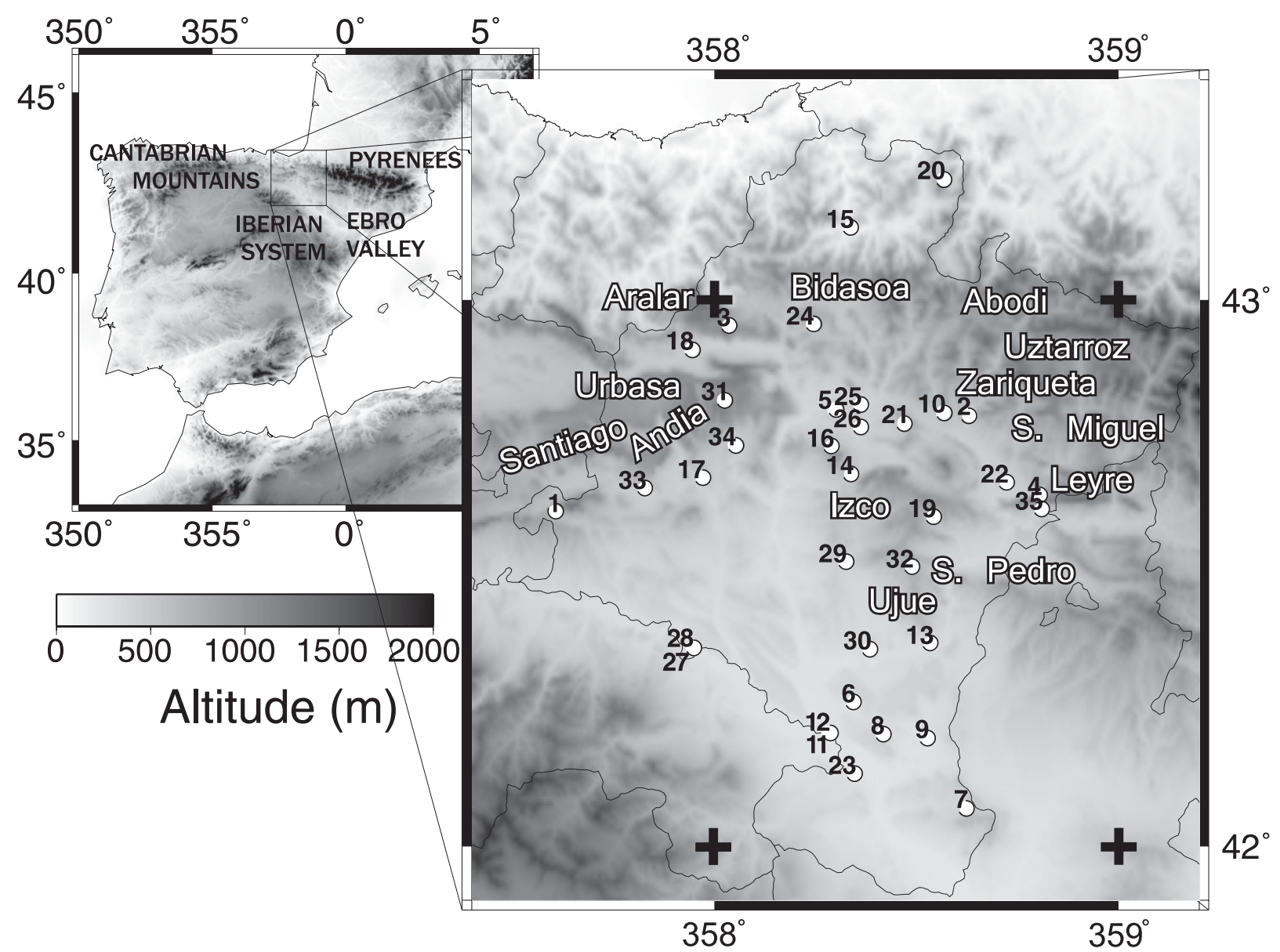

FIG. 1. Location of the CFN within the Iberian Peninsula and meteorological stations (circles). (left) The most relevant geographical features of the Iberian Peninsula surrounding the CFN, and (right) some regional details within the CFN. Shading represents altitude. In the right panel, the thin lines represent political boundaries and the crosses indicate the locations of the ECMWF reanalysis/analysis data closer to CFN.

models. Typically, short temporal periods of a few months or specifically the duration of an observational campaign are simulated to limit the computational requirements (Zhong and Fast 2003; Cairns and Corey 2003; Rife et al. 2004; Zagar et al. 2006). Although the computational power reached in the last years makes multiyear simulations feasible (Buckley et al. 2004; Conil and Hall 2006; Walter et al. 2006), long simulations at higher spatial resolutions that better capture the details of complex orography and allow for evaluations of variability at longer time scales are still computationally demanding and thus have not been so far extensively used.

First, this study explores the accuracy of the mesoscale simulation to reproduce the wind at each observational site to analyze representativeness errors and subsequently compare local versus regional evaluations. The regional series are obtained by averaging the wind at the sites within each region identified by JEA08. The use of average regional series damps local effects in the observations as well as random representativeness errors in the simulation; thus, it provides a first framework to evaluate the numerical simulation performance. Wavelet analysis (Foufoula-Georgiou and Kumar 1995; Torrence and Compo 1998) is used to compare the spectral densities of the observed/simulated regional time series. Additionally, a complementary evaluation is obtained by applying the same regionalization methodologies used by JEA 08 to the simulated wind and comparing the results with those obtained in the observational analysis. To provide comparable classifications, the regionalization methodologies are applied to a subset of the WRF simulation that replicates in detail the temporal and spatial availability of observations along the restricted 1999-2002 period used in JEA08. By doing so, we are able to determine the ability of the WRF simulation in reproducing the subregions found in the observational assessment. 
As discussed earlier, the distribution of wind sensors over a region of interest and the availability of observations through time is an issue of relevance that conditions our understanding of the variability of any meteorological observable. To investigate the impact that the lack of observations over some areas of the CFN can have on the analysis of surface wind variability, the WRF simulation is used as a tool to estimate the likely behavior of the wind field in areas and time intervals with scarcity of observations. This inference analysis is accomplished in two steps: first, by applying the regionalization methodologies to the simulation over the complete observational period (i.e., 1992-2005 instead of 1999-2002); second, by considering the full simulated domain over the CFN (8640 grid points) instead of only the nearest grid points to available observational stations. This allows the evaluation of the extent to which the limited temporal and spatial coverage of the observational network is appropriate to represent the wind variability over the area. Therefore, the results of this work are useful for the design of observational networks.

\section{Observational dataset and WRF simulation}

The wind dataset used by JEA08 to analyze the wind variability over the region consisted of the 35 stations with the best data quality in the CFN meteorological network (Fig. 1); measurements were available from 1 January 1992 to 30 September 2002. The present study uses the same 35 sites, with observations extended until 7 October 2005. The wind speed and direction were recorded as an integrated average for every 10 -min interval, except for seven stations that after 2004 recorded 30-min intervals. The observations were transformed to daily averages of meridional and zonal components.

The mesoscale model used is the WRF modeling system (version 2.1.2; Skamarock et al. 2005). The experiment is configured with four domains using two-way nesting to reach a horizontal resolution of $2 \mathrm{~km}$ over the CFN (Fig. 2). The outermost domain is centered on the CFN and has a size of approximately $2000 \times 2000 \mathrm{~km}^{2}$ and a horizontal resolution of $54 \mathrm{~km}$. The other domains are nested to progressively reach the desired resolution of $2 \mathrm{~km}$ in the innermost domain imbedding the whole CFN. The topographic data are obtained from the U.S. Geological Survey (USGS) global 30 arc-s elevation (GTOPO30) dataset (Bliss and Olsen 1996; Gesch and Larson 1996; Verdin and Greenlee 1996). The default WRF configuration with 31 terrain-following hydrostatic pressure levels (Laprise 1992) was used in the vertical for all domains, with the top level being located at $50 \mathrm{hPa}$.

The longwave and shortwave radiation schemes are based on Mlawer et al. (1997) and Dudhia (1989), re- spectively. A modified version of the Kain and Fritsch (1990, 1993) scheme is employed for the cumulus parameterization in the three outermost domains (absent in the innermost domain). The Yonsei University (YSU) PBL parameterization (Hong et al. 2006) is used in the four domains. With respect to the microphysics, the WRF single-moment six-class scheme, which is similar to Lin et al. (1983), is adopted. Finally, a simple five-layer land surface model based on Dudhia (1996) is used. The USGS land use/land cover system with a horizontal resolution of $1 \mathrm{~km}$ is used to determine the surface physical properties (Anderson et al. 1976).

Initial and boundary conditions were obtained from the 40-yr European Centre for Medium-Range Weather Forecasts (ECMWF) Re-Analysis (ERA-40; Simmons and Gibson 2000; Uppala et al. 2005) from 1992 to August 2002. Afterward, the operational ECMWF analyses are employed. The horizontal resolution of both datasets is $1^{\circ}$ latitude $\times 1^{\circ}$ longitude. The model is initialized as a "cold start" at 0000 UTC each day and run for $48 \mathrm{~h}$, updating the boundary conditions every six hours and recording data every hour. The first $24 \mathrm{~h}$ are discarded as model spinup, retaining the outputs for the following $24 \mathrm{~h}$ and calculating the average of the meridional and zonal wind components at $10 \mathrm{~m}$ above ground level to obtain the daily-mean surface wind field. The process was repeated for the whole observational period: 1 January 1992-7 October 2005. This sequence of short runs with numerous reinitializations has been shown to outperform long-term continuous simulations with only one initialization (Pan et al. 1999; Qian et al. 2003; Lo et al. 2008) and is becoming increasingly accepted and adopted in recent years (Conil and Hall 2006; Zagar et al. 2006).

\section{Methods and rationale}

\section{a. Background: Regionalization methodologies and previous results}

JEA08 performed the wind regionalization with two methodologies based on principal component analysis (PCA; Preisendorfer 1988; von Storch and Zwiers 1999). The first one identifies the subregions by grouping the sites with similar loads on their principal modes using cluster analysis (CA). A two-step CA approach was adopted (Milligan 1980): in the first step, the hierarchical complete linkage algorithm was used to decide the number of clusters (subregions); in the second step, the nonhierarchical $K$-means scheme was used to reorder the stations. The second methodology rotates the principal modes to isolate the subregions using the varimax scheme. The main difference between the classifications 


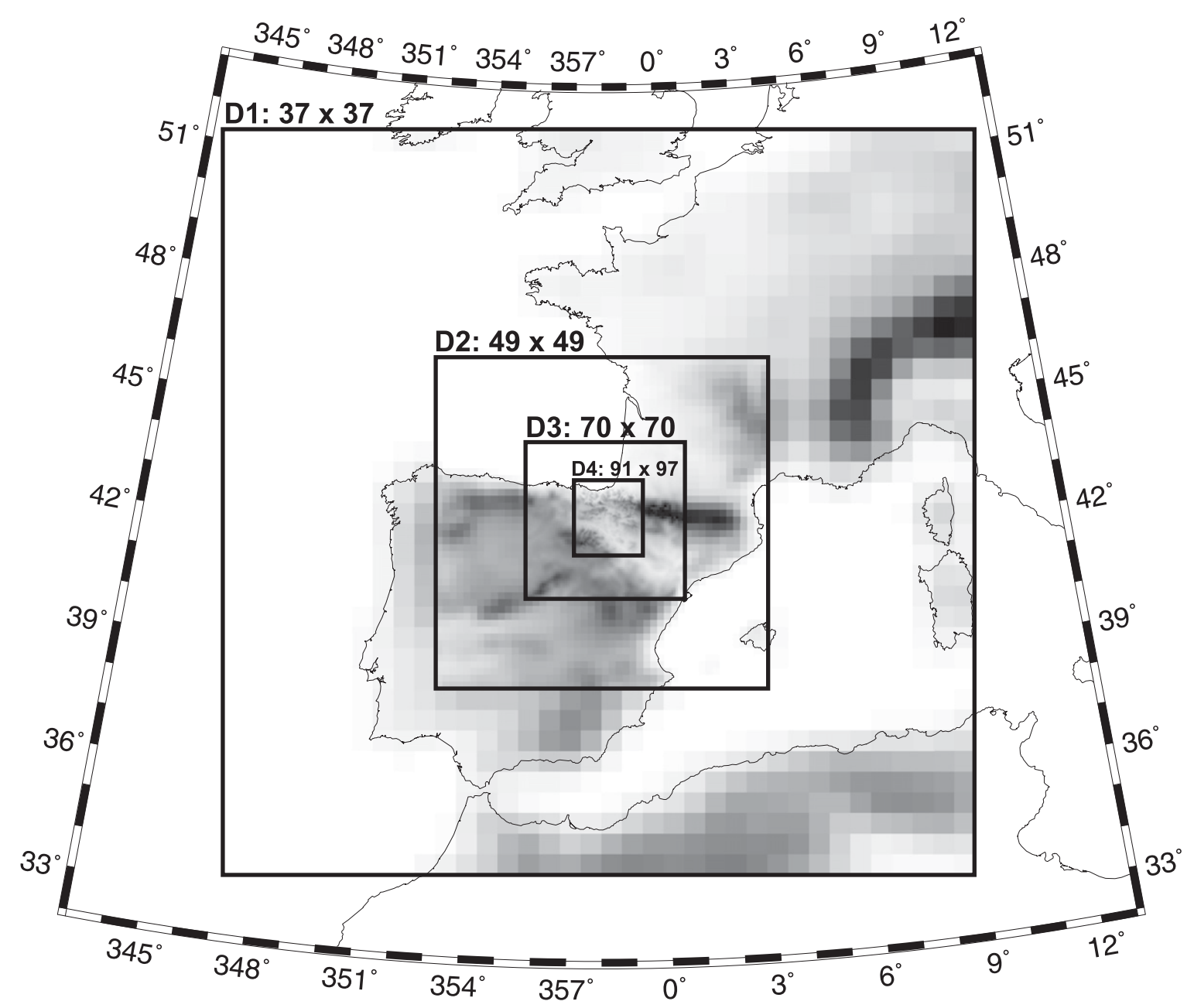

FIG. 2. Spatial configuration of domains used for the numerical simulation: four domains two-way nested with $54,18,6$, and $2 \mathrm{~km}$ of horizontal resolution. The orography of each domain is displayed according with their particular resolution. The number of grid points of each domain (west-east by north-south) is also displayed.

is that the rotational procedure allows for overlapping subregions.

Because the existence of missing data in the observational series could potentially have an impact on the PCA results, a reduced subset of 769 daily-mean wind fields with a good spatial representation (more than $80 \%$ of the stations available) and a homogeneous distribution through the year was used for the identification of the subregions. The so-called reduced dataset spanned 1999-2002.

The CFN wind subregions obtained in JEA08 with both methodologies are reproduced in Fig. 3 (modified from JEA08) for the sake of comparison with model results. Both regionalizations identified similar groups of stations: a subregion in the Ebro valley (EV; circles); a group mainly formed by the mountain stations (MS; squares); several groups of stations with a north-south orientation were identified by the CA approach (hexa- gons, pentagon, and diamonds in Fig. 3a), whereas the rotational procedure integrated them into one subregion (NS; diamonds in Fig. 3b); and a fourth subregion formed by stations in the narrow valleys to the north of the Ebro basin (NV; triangles). Particularly relevant for this study was the finding that the meridional wind variability is rather similar in all the emergent subregions, with the zonal wind variability being responsible for the differences between them. The EV and NV subregions present similar zonal variability, probably because of the rather parallel orientation of their valleys, but they were kept as independent subregions in JEA08 because of their discrimination by the two regionalization approaches and their location at two different valleys.

\section{b. Evaluation of the WRF numerical simulation}

The performance of the WRF simulation is first evaluated at the local scale by comparing the observed and 


\section{OBSERVATIONS}
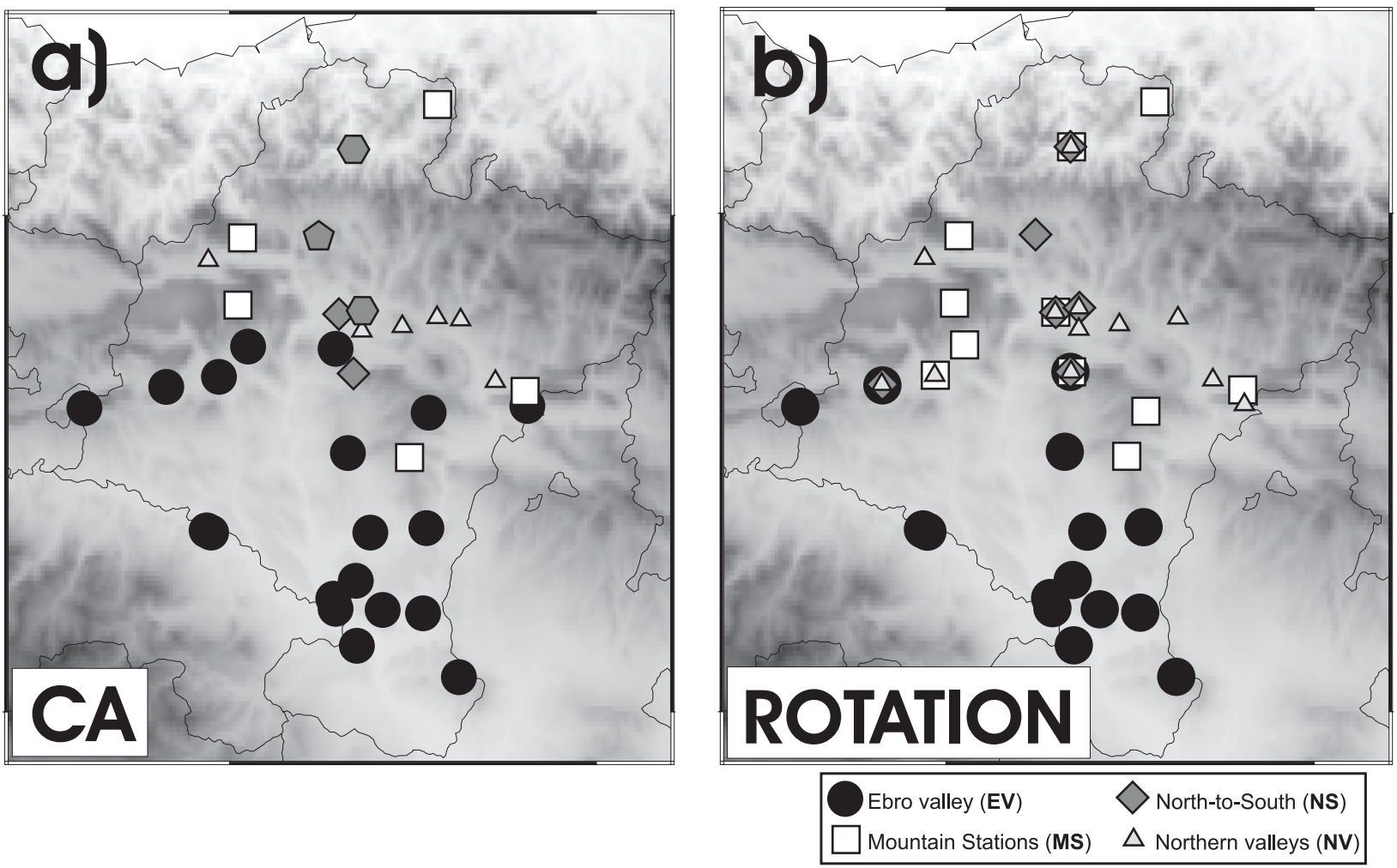

FIG. 3. Wind regionalization obtained by JEA08 after applying the methodology based on (a) cluster analysis and (b) rotation of the principal modes to the observational reduced dataset that spans the 1999-2002 period (Table 1). Locations with the same symbol indicate that the sites present similar temporal wind variability and therefore define a subregion. The regionalization of (b) is used in this work to calculate the averaged time series at each subregion (see the key).

simulated variability at the individual stations. By doing so, we will illustrate the advantages of the regional approach adopted here and the added value of the regional simulation with respect to the original reanalysis/ analysis fields.

Second, the performance at the regional scale is evaluated with two independent analyses. The first evaluates the ability of the simulation to reproduce the temporal wind variability at the subregions identified in JEA08. The second approach applies the regionalization methodologies to the simulated wind, thus allowing for the evaluation of the ability of the simulation to replicate the spatial distribution of observed subregions. Wavelet analysis is applied to explore the observed and simulated variability in the frequency domain.

Because the WRF simulation provides a higher spatial and temporal resolution than the observational network, it is necessary to manipulate the simulation to provide comparable datasets. Two simulated datasets are created to replicate the spatial and temporal distribution of observations at the 35 sites (Fig. 1):
- The spatially and temporally masked simulation (STMS) consisting of the simulated wind at each site as represented by the nearest grid point: Here, spatial masking refers to the fact that only the 35 nearest grid points to the observational sites are used for analysis, whereas the temporal mask means that the simulated values corresponding to a missing observation are suppressed. This WRF dataset spans the complete observational period from 1992 to 2005.

- The reduced spatially and temporally masked simulation (reduced STMS), which is comparable to the reduced observational dataset in JEA08 and also consists of the same 769 days over the period 1999-2002 used to identify the observational subregions.

The ability of the numerical simulation to reproduce the wind variability over the CFN will first be evaluated by comparing the observed and simulated temporal wind variability at the different observational subregions. The grouping of stations obtained with the methodology based on the rotational procedure (Fig. $3 b$ ) is used to 
define the subregions. Though both are comparable, the rotational approach does not produce single station groups (Fig. 3a), a desirable feature to emphasize the regional evaluation. "Regional time series" refers to the time series resulting from averaging the series from the stations belonging to each of the subregions isolated with the rotational procedure (Fig. 3b).

This approach allows for an evaluation of the performance of the simulation in the observational regions. However, the question remains open as to whether the model would provide a similar classification if the same regionalization analysis performed on observations was carried out for the WRF simulation.

Therefore, a complementary evaluation of the WRF simulation will be attained by applying the two different regionalization approaches to the reduced STMS in the simulation and comparing results with those from observations (Fig. 3). Despite the relatively high resolution, such comparison is still subject to the effects of representativeness errors and local features that are not captured by the model. A detailed agreement between regionalizations is therefore not to be expected, although one would hope to find similarities between their main characteristics.

\section{c. Inference analyses: The effects of observational sampling}

On the basis of the accuracy displayed by the simulation during the evaluation phase, the simulation is subsequently used to address the impact that the limited temporal and spatial coverage of observations may produce on the analysis. With this aim, the wind regionalization methodologies are applied avoiding temporal and/or spatial masking. Additional subsets of the simulated wind for the specific purposes of the inference analysis are created:

- The spatially masked simulation (SMS), which consists of the simulated wind nearest each of the 35 observational sites: It includes no temporal masking, thus spanning the entire 1992-2005 simulated period.

- The complete simulation, which consists of the wind series at all the simulated (8640) grid points for the entire 1992-2005 period (i.e., all available information from the WRF simulation).

The regionalization obtained with the SMS dataset will be compared with the regionalization obtained by JEA08 (Fig. 3). This comparison allows the influence of the length of the observational period in identifying the subregions to be analyzed. The influence of the observational spatial coverage in identifying the subregions will be analyzed by performing the regionalization with the complete simulation. These two analyses have im-
TABLE 1. Characteristics of the wind datasets used in the comparison of observations and the WRF simulation.

\begin{tabular}{lcccc}
\hline $\begin{array}{c}\text { Simulated } \\
\text { dataset }\end{array}$ & $\begin{array}{c}\text { Observational } \\
\text { dataset }\end{array}$ & $\begin{array}{c}\text { Temporal } \\
\text { coverage }\end{array}$ & $\begin{array}{c}\text { Time } \\
\text { series }\end{array}$ & $\begin{array}{c}\text { Missing } \\
\text { values }\end{array}$ \\
\hline Reduced STMS & Reduced & $1999-2002$ & 35 & Yes \\
STMS & Complete & $1992-2005$ & 35 & Yes \\
SMS & - & $1992-2005$ & 35 & No \\
Complete & - & $1992-2005$ & 8640 & No \\
\hline
\end{tabular}

plications for the design of observational networks. The temporal coverage and the number of time series in each observational (reduced and complete) and simulated dataset (STMS, reduced STMS, SMS and complete) used in the study are summarized in Table 1.

\section{Results}

\section{a. Local versus regional}

A comparison of the reduced dataset from JEA08 with its equivalent dataset from the WRF simulation (reduced STMS; Table 1) is displayed in Fig. 4, where the correlation (circles) and the root-mean-square error (RMSE; circumferences) between observed and simulated wind are plotted for each site and wind component. The RMSE at each site is calculated with the time series of the anomalies after removing the climatological mean $\bar{x}$ and subsequent normalization by the standard deviation $s_{\mathrm{obs}}$ of the observational time series:

$\mathrm{RMSE}=\frac{\sqrt{\frac{1}{N} \sum_{i=1}^{N}\left[\left(x_{\mathrm{obs}}-\bar{x}_{\mathrm{obs}}\right)_{i}-\left(x_{\mathrm{wrf}}-\bar{x}_{\mathrm{wrf}}\right)_{i}\right]^{2}}}{s_{\mathrm{obs}}}$.

The zonal correlation increases toward the south, with the highest values at stations in the EV and MS subregions (Fig. 4a). The lowest correlated sites, mainly located in the NS and NV subregions, are where the normalized RMSE values are highest (often above one; Fig. 4b). The meridional correlation is high everywhere (Fig. 4c), except at stations 15 and 24 (Fig. 1), where the normalized RMSE values are high (Fig. 4d). The low (high) values of correlation (RMSE) depict the locations of poorer model performance.

A quantification of the influence of representativeness errors is not straightforward, but it can be estimated by comparing the highest correlation and lowest RMSE between the site and the 25 nearest grid points, which is also displayed in Fig. 4. The smallest (largest) RMSE (correlation) among the nearest 25 grid points is represented with a circle (circumference), which shows the 

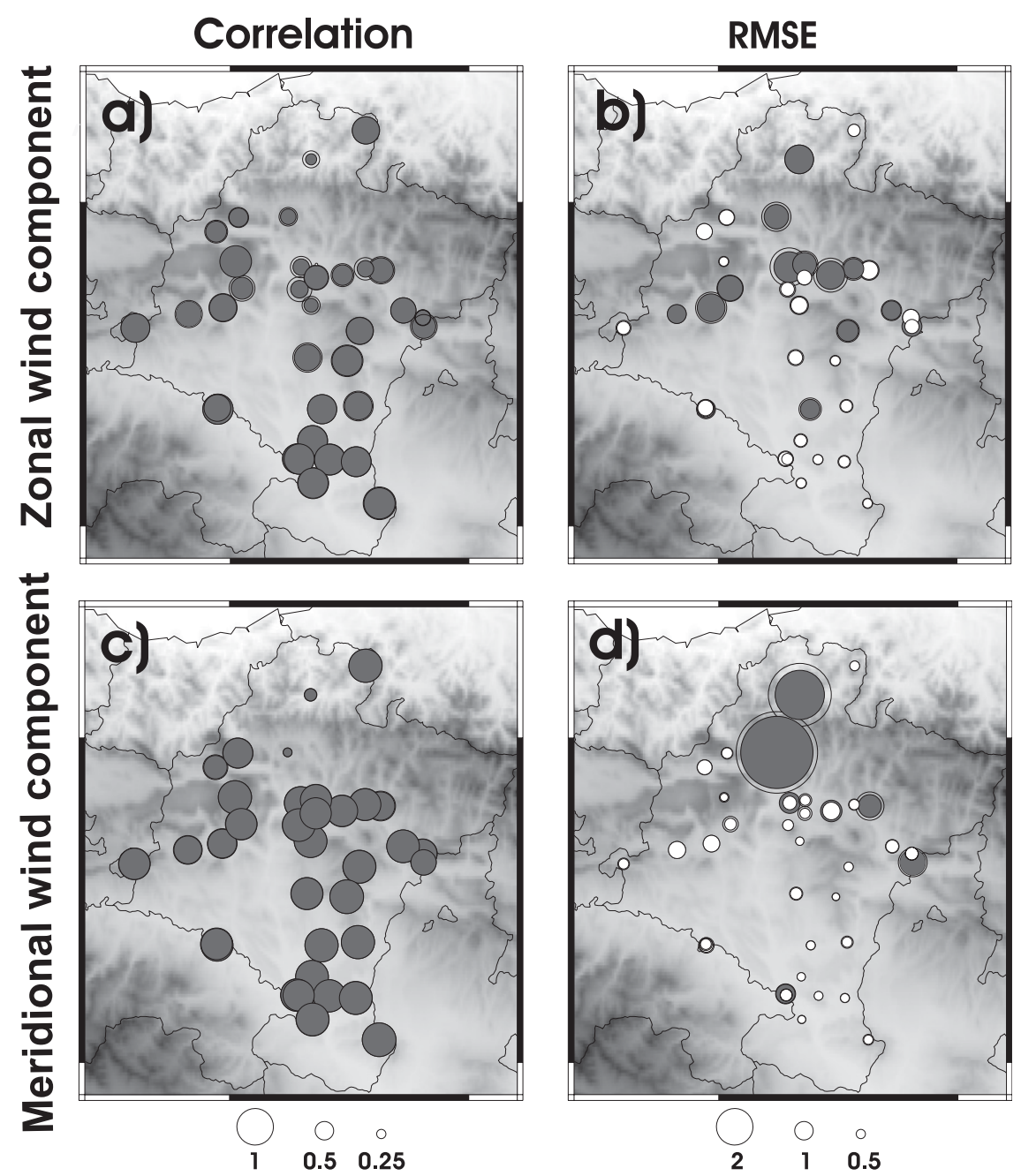

FIG. 4. (a),(c) Correlation and (b),(d) RMSE of the observed and simulated zonal and meridional wind, as described in the text. The inner circle diameters are proportional to the correlation between observations and simulations from the nearest grid point; the outer circumferences radius shows the highest correlation between a site and the nearest 25 grid points. For RMSE [Eq. (1)], the external circumferences are proportional to the RMSE between the site and the nearest grid point; the internal radii represents the smallest RMSE among the nearest 25 grid points. White (gray) circles indicate that the RMSE is lower (higher) than 1.

correlation (RMSE) with more distant grid points only when improved values are found.

The sites with the best RMSE and correlation values do not show better values when compared with more distant grid points (Fig. 4). However, the sites with poorer scores (mostly in the NS and NV subregions) often show better correlation and lower RMSE at some nearby grid point than at the closest one. This indicates that a misrepresentation of the observed variability could be taking place at some locations by assigning observations to the nearest grid point. The reasons for this behavior could be related to the discretization of the orography and/or the surface physical properties. However, the effects of the representativeness errors are small, which indicates that the use of the nearest grid point seems to be a reasonable approximation for this particular case.

It was argued in the introduction that averaging local observations into regional series filters out local variability and enhances the regional signal. Because section $4 \mathrm{~b}$ describes an evaluation of the model performance at the scale of the subregions found in JEA08, it is interesting to illustrate the impact on the simulation performance associated with the change from local scales to subregional averages. The comparison has been performed using Taylor (2001) diagrams. The Taylor diagram used herein [different from that introduced by 
Taylor (1917) and Montgomery (1950)] is a polar plot to compare two time series, usually an observationsimulation pair. The angle depicts correlation ranging from 0 ( $y$ axis) to 1 ( $x$ axis). The radial coordinate represents the ratio of the standard deviations of the two time series (normalized Taylor diagram). Hence, a "perfect" agreement between the observation-simulation pairs reaches a value of 1 over the $x$ axis. Because the standard deviations of two time series and their correlation are related with the RMSE of the anomalies by the law of cosines (see inset in Fig. 5a), the RMSE is also displayed in the diagram [gray isolines centered in $(1,0)]$. Hence, the diagram is useful to summarize the correlation, RMSE of the anomalies, and ratio of the variances between observed-simulated pairs (Taylor 2001).

Figure 5 shows Taylor diagrams (Taylor 2001) calculated with the simulated and observed wind components for each region, both for the regional averages and the individual sites. In most subregions, the averages agree better than when comparisons are made at the site level. A clear example is the zonal wind component of the MS subregion (Fig. 5c) or the meridional wind component of the NS subregion (Fig. 5f). This shows the beneficial effect of averaging the observed and simulated series to mitigate local effects and reinforce the regional signal. The simulation shows a better performance reproducing the wind variability in EV and MS subregions than in NV or NS. This last subregion shows the worst performance.

Finally, the added value of the WRF simulation over ECMWF results is determined comparing both models wind against observations at the four subregions (Fig. 6). In the case of the WRF simulation, the regional wind components are calculated with the reduced STMS (Table 1), as in Fig. 5. Hence, there is a simulationobservation pair for each subregion (solid symbols). In the case of the ECMWF, the daily averaged surface wind components at the four grid points closest to the CFN (Fig. 1) are compared with the regional wind components at each subregion. Therefore, there are four observation-simulation pairs for each subregion (hollow symbols), because it is impossible to obtain a time series for each subregion with the coarse spatial resolution of the ECMWF data $\left(1^{\circ}\right)$.

Both models reproduce more satisfactorily the meridional wind than the zonal wind (Fig. 6a,b). This is not surprising, given the results in Figs. 4 and 5. The reason for this may be the location of the CFN between the two northern Iberian mountain systems (i.e., Cantabrian and Pyrenees) that strongly modify surface circulations, blocking and channeling the flow between them and probably producing similar meridional wind variability over the whole CFN (JEA08). The mountain systems are large enough to be represented in the simulation, even with the coarser ECMWF spatial resolution (more than $100 \mathrm{~km}$ ), so the ECMWF data capture the meridional wind variability introduced over the $\mathrm{CFN}$ as a consequence of the surface flow channeling. For the meridional component (Fig. 6b), correlation values between either WRF or ECMWF and the observations are around 0.9 and the RMSE is below 1.0. The WRF simulation slightly increases the correlation shown by the ECMWF data (Fig. 6b) with a tendency to increase variability, leading to overestimation in the NS and NV subregions. There is better agreement with observations in the MS and EV subregions.

The WRF model statistics compare better with the observational zonal component (Fig. 6a), especially for the EV and NV subregions, where correlations increased from 0.2 to 0.85 and from 0.4 to 0.8 , respectively. The best agreement between ECMWF data and observations in the case of the zonal component was in the MS subregion. Because the zonal wind variability causes the differences between the subregions, it can be expected that the inclusion of a more realistic orography will substantially impact the accuracy of its simulation. In this way, the coarser ECMWF horizontal resolution is not able to resolve the CFN mountain ridges leading to low correlation at the valley subregions and better scores for the MS subregion. WRF's higher spatial resolution improves representation of the orography and the simulation of the surface zonal component variability at the valley subregions.

This interpretation is purely dynamical. It neglects the thermal forcing that may also be better represented by a mesoscale model, contributing also to the better scores shown by WRF.

\section{b. Evaluation: WRF replication of observed regional variability}

In the following section, WRF's ability to reproduce the regional wind variability is measured by 1) comparison of the temporal wind variability in the subregions identified by JEA08 and 2) comparisons of the regionalization methodology results applied to simulated and observed winds.

\section{1) EVALUATION OVER OBSERVATIONAL REGIONS}

Table 2 gives correlation, bias, and normalized RMSE for the STMS datasets in Table 1. That is, the entire 19922005 period is used in the comparison. These scores agree well with those displayed in Fig. 6 for the reduced datasets (1999-2002). This extends the previous interpretations to the whole observational period. To further illustrate this statement, the regional time series of the 


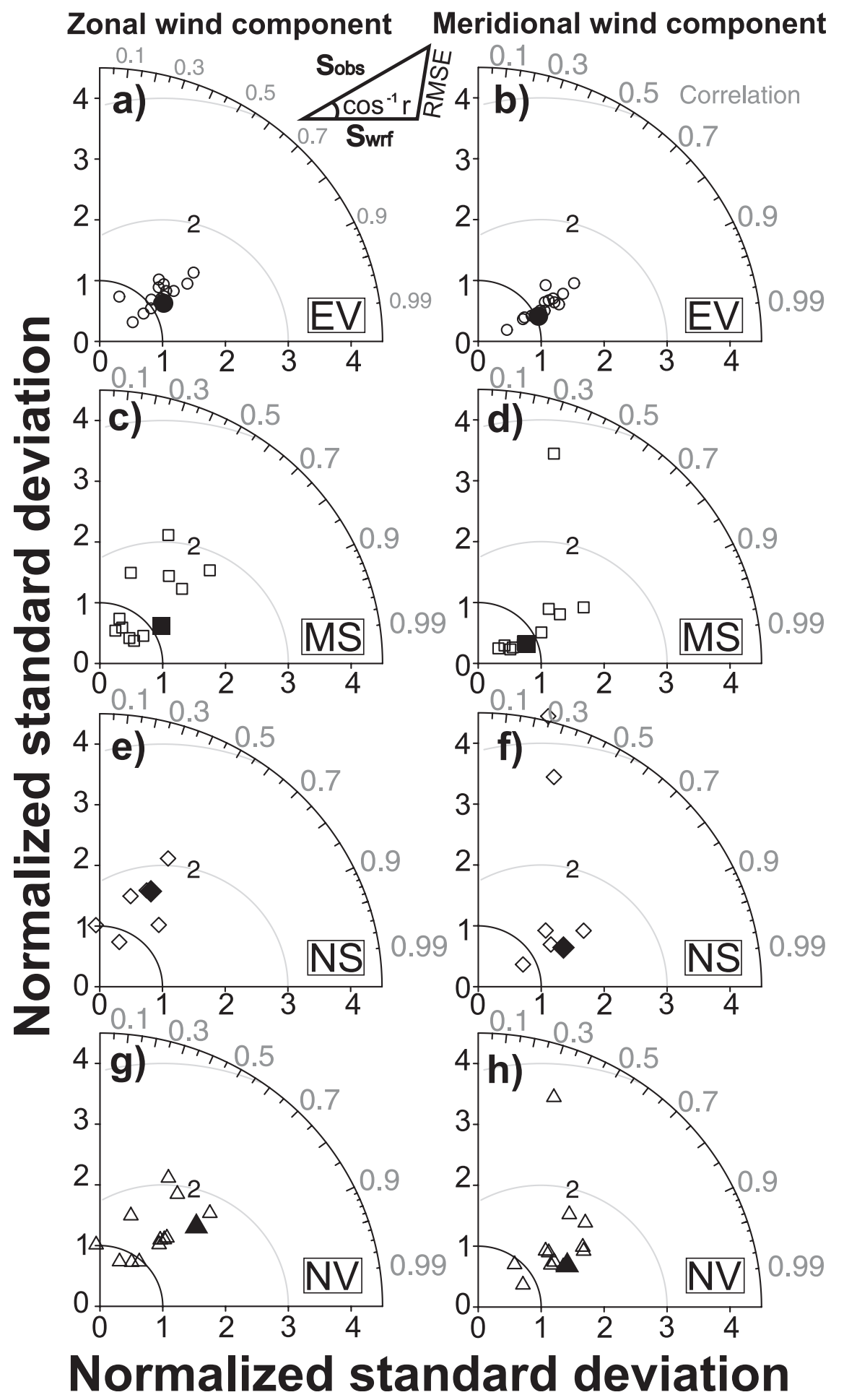

FIG. 5. Normalized Taylor diagrams comparing the (left) zonal and (right) meridional wind components at each of the four subregions identified with observations (Fig. 3b). The hollow symbols represent the comparison at each observational site, whereas the solid symbols represent the comparison of the regional time series. The reduced datasets are used in the comparison (Table 1). 


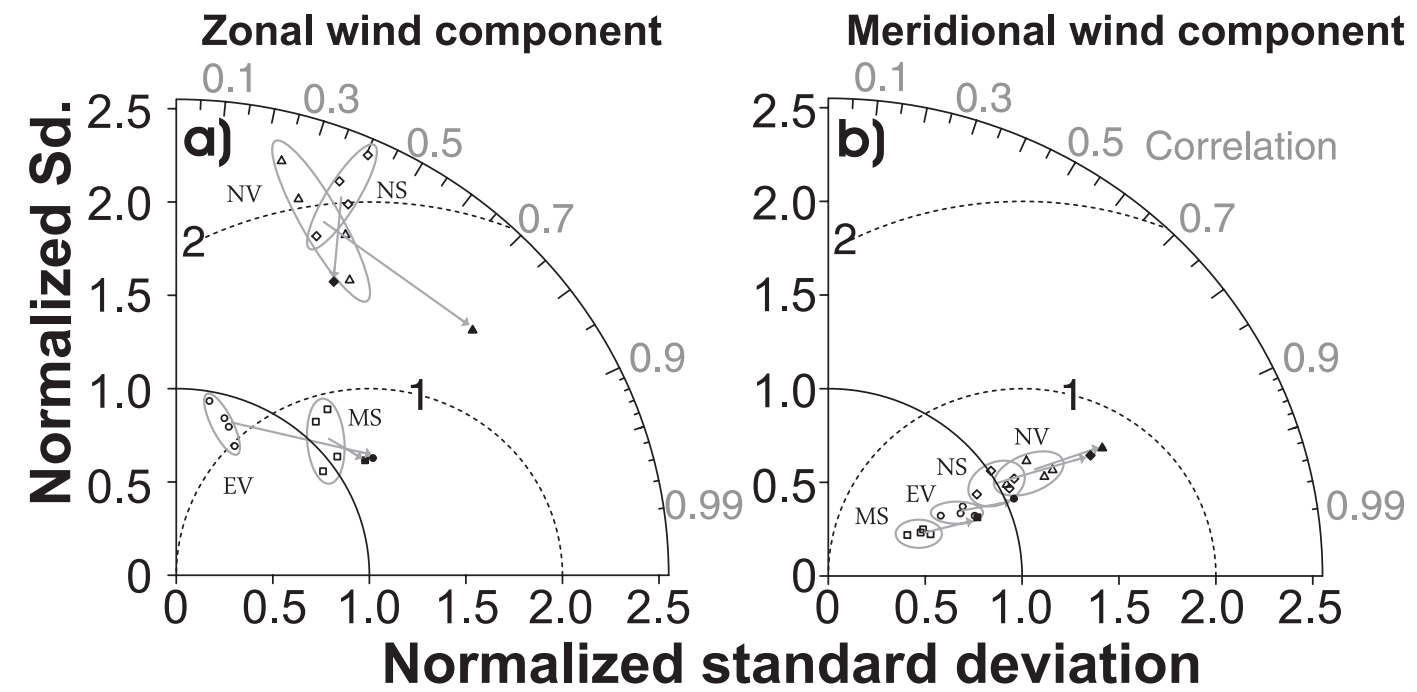

FIG. 6. Normalized Taylor diagrams comparing the daily-mean (a) zonal and (b) meridional wind components from the WRF simulation (solid symbols) and the ECMWF reanalysis/analysis (hollow symbols) with the observed wind at each of the four subregions (Fig. 3b): EV (circles), MS (squares), NS (diamonds), and NV (triangles). The ellipses enclose the comparison of the observations at a given subregion and the ECMWF simulations at the four closest grid points to the CFN (see zoomed area in Fig. 1). The arrows highlight the different performance displayed by the ECMWF and the WRF simulation. The comparison is restricted to the 769 days of the reduced datasets.

zonal component from the STMS and the complete observational datasets (Table 1) are displayed in Fig. 7. This component has been selected for illustration, because it is responsible for the different wind variability of the subregions (JEA08). The series show similar temporal variability within and outside the reduced period, in concordance with the similar scores found in Fig. 6 and Table 2. The time evolution of the RMSE is also displayed in Fig. 7. There is a tendency toward lower RMSE values during summer than winter, revealing a certain seasonal behavior in the performance of the numerical simulation. At the EV, MS, and NV, the numerical experiment is insensitive to the station number, but it has some relevance for the NS subregion, which always had few stations, particularly before the year 2000 .

To avoid introducing potential biases by constructing regional time series from a variable number of stations (Fig. 7) with different variances, each time series was standardized by subtracting its mean and dividing by its standard deviation before calculating the regional average. The correlation between these observed and simulated regional time series $(r=0.85,0.85,0.52$, and 0.76 for EV, MS, NS, and NV, respectively) are similar to those obtained with the averaged raw series (Fig. 7). This indicates that the regional signal is neither degraded nor improved with the standardization and therefore is not subject to the dominant variability of a few sites.

Complementary information to evaluate the performance of the simulation in reproducing the observed temporal variability is obtained through a Morlet wavelet analysis (Foufoula-Georgiou and Kumar 1995; Torrence and Compo 1998). These are shown for the observed and simulated regional zonal component of the EV and MS subregions in Fig. 8. The EV shows high similarities between the observed and simulated power spectra (Figs. 8a,c, respectively). It shows activity at the highest frequencies throughout the whole observational period. The lowest frequencies reflect an alternation of significant contributions to the annual cycle. The MS, on the contrary, shows observational wavelet spectra with a significant annual band during the whole observational period (Fig. 8b). The simulation reproduces partially the density around the annual band, but not as continuously as in the observations (Fig. 8d). The observed reduction in variance after the year 2000 for periods between 200 and 20 days is captured by the simulation. This reduction may be caused by the varying number of stations available to calculate the regional time series (Fig. 7).

TABLE 2. Correlation, bias (observations - simulations), and RMSE [Eq. (1)] calculated with the regional time series of the zonal/meridional wind components from the complete observational dataset and the STMS (Table 1).

\begin{tabular}{lccc}
\hline \hline & $\begin{array}{c}\text { Correlation } \\
u / v\end{array}$ & $\begin{array}{c}\text { Bias } \\
u / v\end{array}$ & $\begin{array}{c}\mathrm{RMSEa} / \mathrm{Sd}_{\text {obs }} \\
u / v\end{array}$ \\
\hline $\mathrm{EV}$ & $0.86 / 0.91$ & $0.46 /-0.72$ & $0.62 / 0.43$ \\
$\mathrm{MS}$ & $0.86 / 0.91$ & $-0.24 /-0.86$ & $0.52 / 0.43$ \\
$\mathrm{NS}$ & $0.54 / 0.86$ & $-0.48 /-0.87$ & $1.31 / 0.63$ \\
$\mathrm{NV}$ & $0.74 / 0.86$ & $-0.21 /-0.31$ & $1.27 / 0.71$ \\
\hline
\end{tabular}



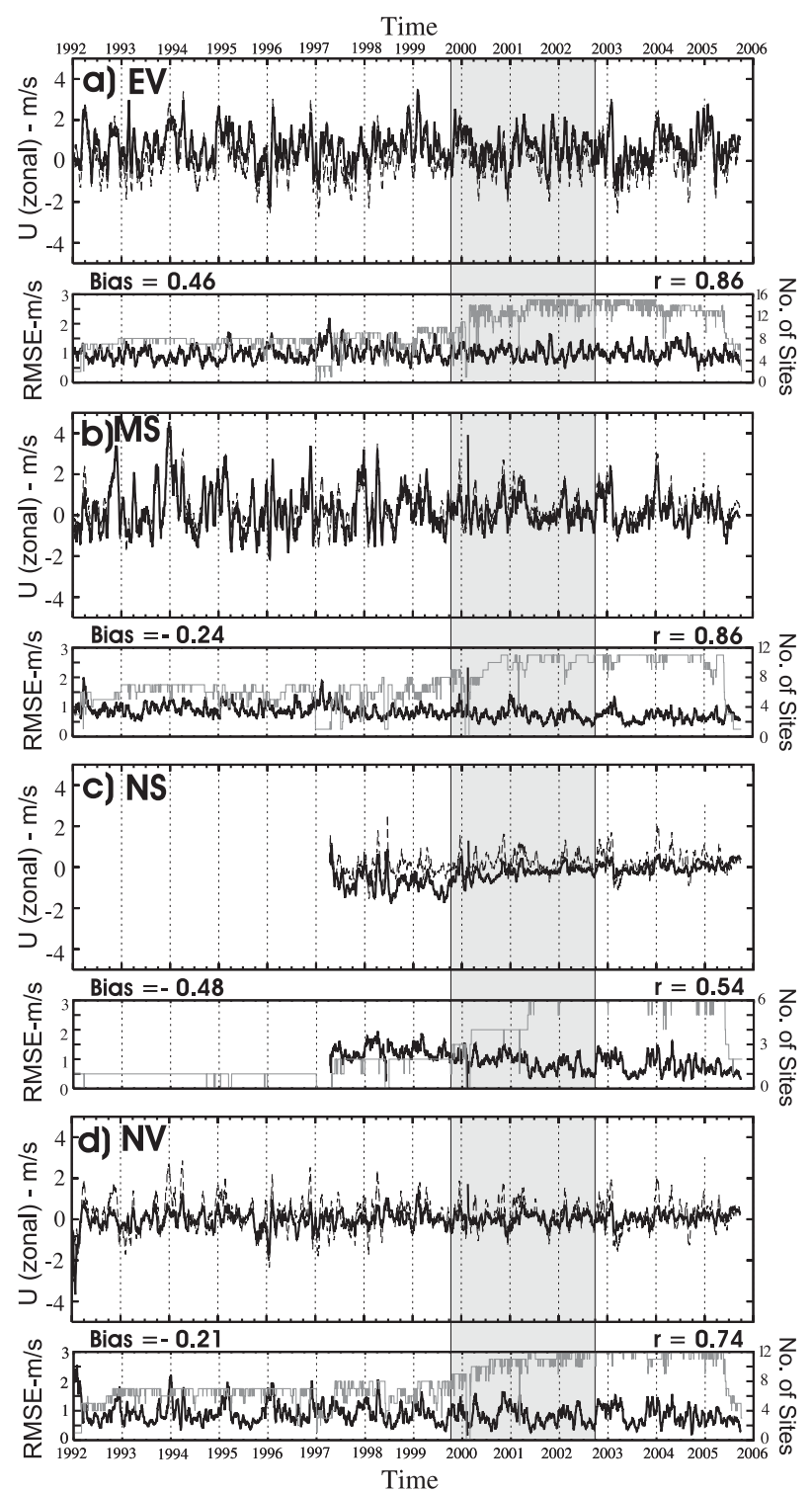

FIG. 7. The top graphs for (a)-(d) show the 20-day moving averages of zonal wind in the (a) EV, (b) MS, (c) NS, and (d) NV subregions (Fig. 3b) calculated with the complete observational dataset (solid lines) and the STMS (dashed lines). The correlation and the bias (observed minus simulated mean) of the regional series before applying the moving average are also displayed. The bottom graphs for (a)-(d) represent the 20-day moving-average RMSE (black lines) and the number of available stations at each particular day (gray lines). The gray area highlighted on the time series represents the time span of the reduced datasets (Table 1).

The extent to which the wavelet power spectra are sensitive to this limitation was analyzed using the complete time series from the numerical simulation (SMS; Table 1). The resulting wavelet power spectra are displayed for the EV and MS subregions in Figs. 8e,f, respectively. Both spectra resemble those calculated with a variable number of stations. This points to a real reduction in regional variability being responsible for the decay in variance observed during the last years (Fig. 8b). The wavelet power spectra of the zonal wind component at the NS and NV subregions also agree with the simulation (not shown), but with some degradation evident in the NS subregion, as expected from fewer time series (Fig. 7c) and a worse representation of wind variability at two of its sites (Fig. 4).

Meridional wind variability is very homogeneous over the CFN, so their regional wavelet power spectra are similar in all the subregions, with similar structure to that displayed for the zonal wind of the EV subregion (Fig. 8a), wherein both components present equivalent variability (JEA08). Better agreement between the wavelet spectra for observations and simulations is found for this component, because the simulation reproduces the meridional component more accurately (Table 2).

\section{2) Regionalization IN THE WRF Simulation}

The regionalization methodologies used by JEA08 to identify the wind subregions (see section 3a) are applied to the WRF simulation. The reduced STMS dataset provides data comparable to those used to identify the observed subregions (Table 1).

The first step in both regionalization methodologies is a PCA (Preisendorfer 1988; von Storch and Zwiers 1999). The regionalization based on the rotation of the main modes of variation identifies one subregion from each retained mode; because the purpose of this analysis is to evaluate the accuracy of the numerical simulation to reproduce the spatial coverage and the wind variability of the subregions identified with observations (EV, MS, NS, and NV), a total of four principal modes are retained. This is in agreement with the CA methodology for which identified subregions do not depend on the number of principal modes retained (JEA08).

The classification obtained with the CA methodology is displayed in Fig. 9a. Overall, the regionalization obtained (Fig. 9a) is similar to that obtained with observations (Fig. 3a), with some differences in the boundaries between subregions. Such discrepancies were expected, because the observations are affected by local effects and the simulation suffers from representativeness error.

The regionalization obtained with the rotational procedure (Fig. 9b) shows, as does Fig. 3, the overlapping classifications that are in contrast to the more rigid CA method. The EV valley subregion (circles) includes most of the same stations as in Fig. 3b. The MS subregion (squares) is also identified as including most of the sites of its observational counterpart. Another subregion (triangles) mainly associated with the NV is identified 

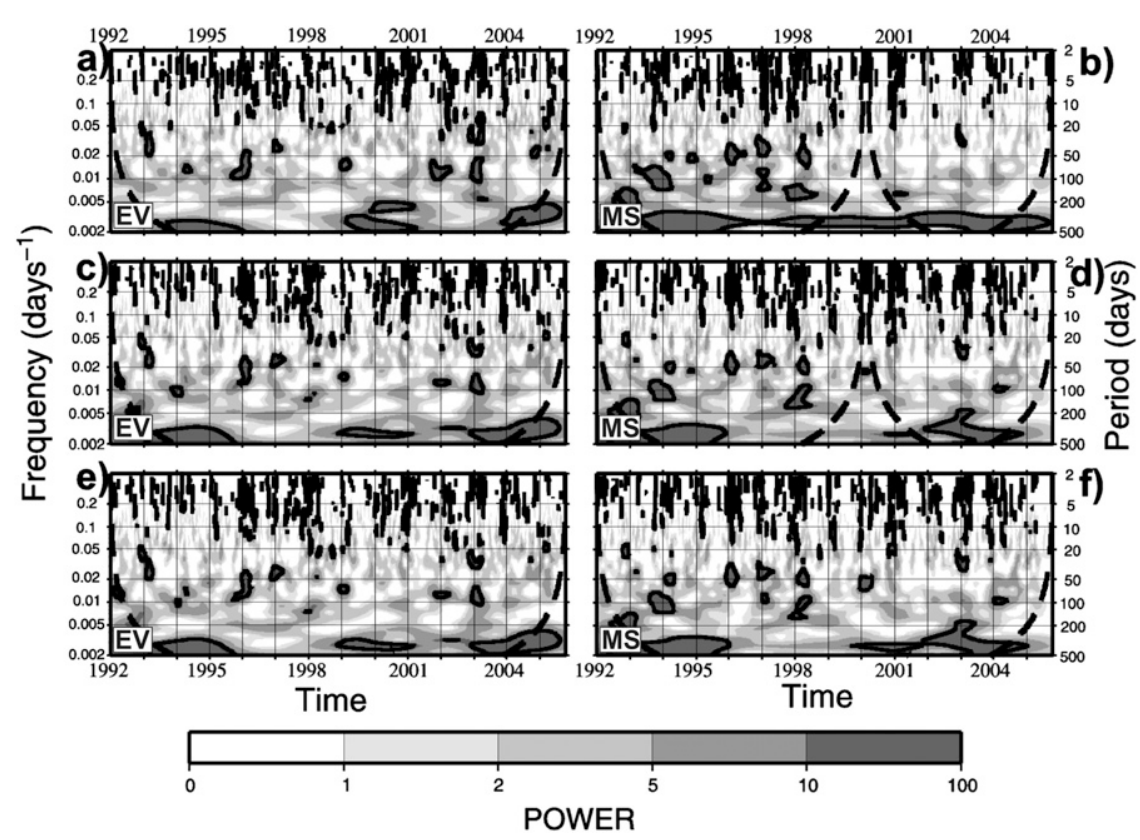

FIG. 8. Wavelet spectral power of the zonal wind component time series for the (left) EV and (right) MS subregions for (top) the complete observational dataset, (middle) the STMS dataset, and (bottom) the SMS dataset. The shaded areas represent normalized variances higher than 1 , 2,5 , and 10 , whereas the black thick contour lines enclose regions of confidence above $95 \%$ for a first-order autoregressive process. The dashed line represents the "cone of influence," below which edge effects become important (Torrence and Compo 1998).

and includes some stations from the Ebro valley. An additional cluster is isolated (diamonds) that includes sites from the observational NS and NV groups. Because its principal component showed slightly higher and sig- nificant correlation values with the observational NS principal component, it was classified as such in the simulation. The regionalization in Fig. $9 \mathrm{~b}$ is similar to that with the observed surface wind (Fig. 3b). The main

\section{Reduced STMS}

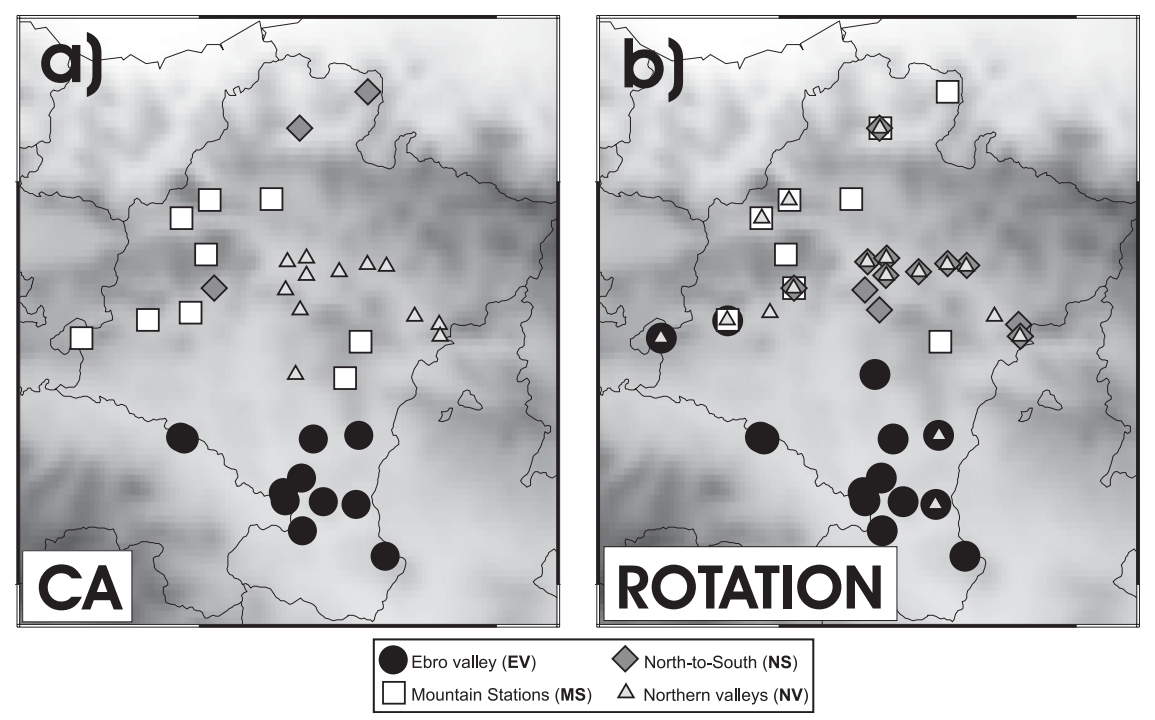

FIG. 9. Regionalization configurations attained after applying the methodology based on (a) the CA and (b) the methodology based on the rotation of the principal components to the reduced STMS dataset (Table 1). The different symbols represent locations with similar temporal wind variability that define the identified subregions. 

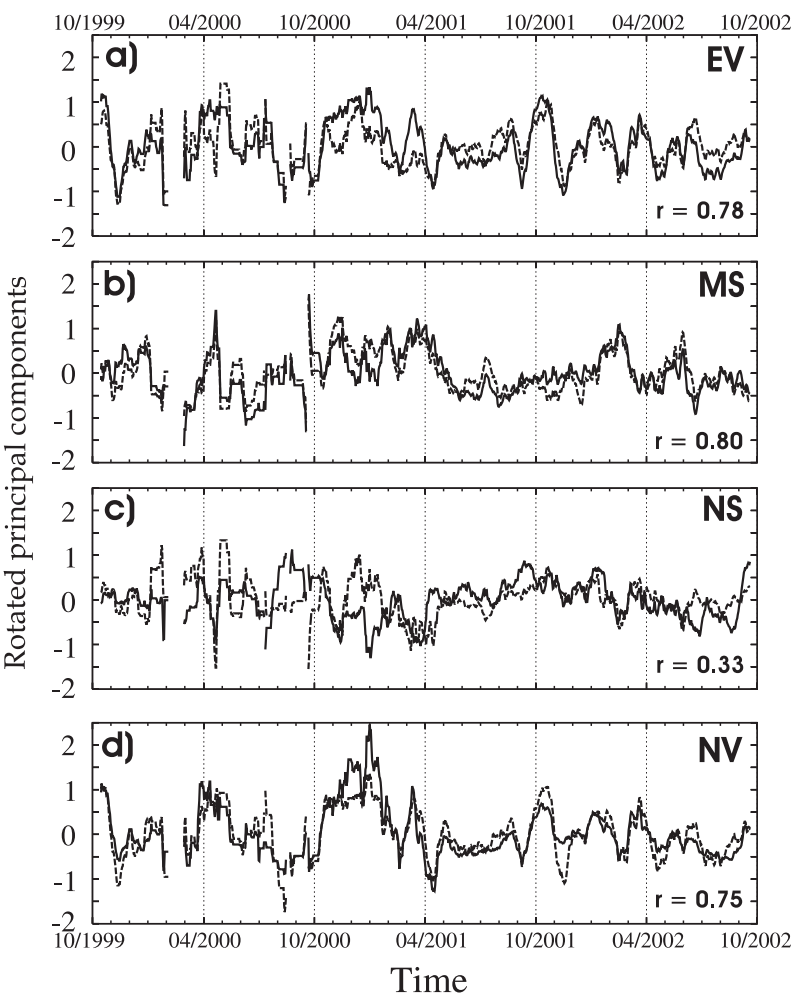

FIG. 10. The 20-day moving-average filter outputs of the rotated principal components associated with the (a) EV, (b) MS, (c) NS and (d) NV subregions. The solid lines are associated with the calculations performed with the reduced observational dataset (from JEA08), whereas the dashed lines are associated with the reduced STMS dataset (Table 1). The correlation between the unfiltered time series at each particular subregion is also shown.

difference is the enlargement of the NS subregions with stations from the NV subregion. The identification problems in the NS subregion are not surprising, given that region's poorer scores (Fig. 6).

Because the rotation methodology assigns one principal mode of variation to each subregion, rotated principal components provide information of the temporal wind variability at the subregions (JEA08). The rotated principal components in the simulation are compared in Fig. 10 against their counterparts in the observations to evaluate the performance of the simulation in reproducing the temporal wind variability at the subregions. Correlation values are over 0.75, except for the NS subregion, which shows a rather low correlation score among the observed and simulated principal components $(r=$ 0.33 ) but improves after the year 2000, when there are more stations reporting.

Complementary information about temporal variability at the subregions can be provided by spectral analysis of rotated principal components. The reduced STMS spans 1999-2002 with many missing data, so wavelet

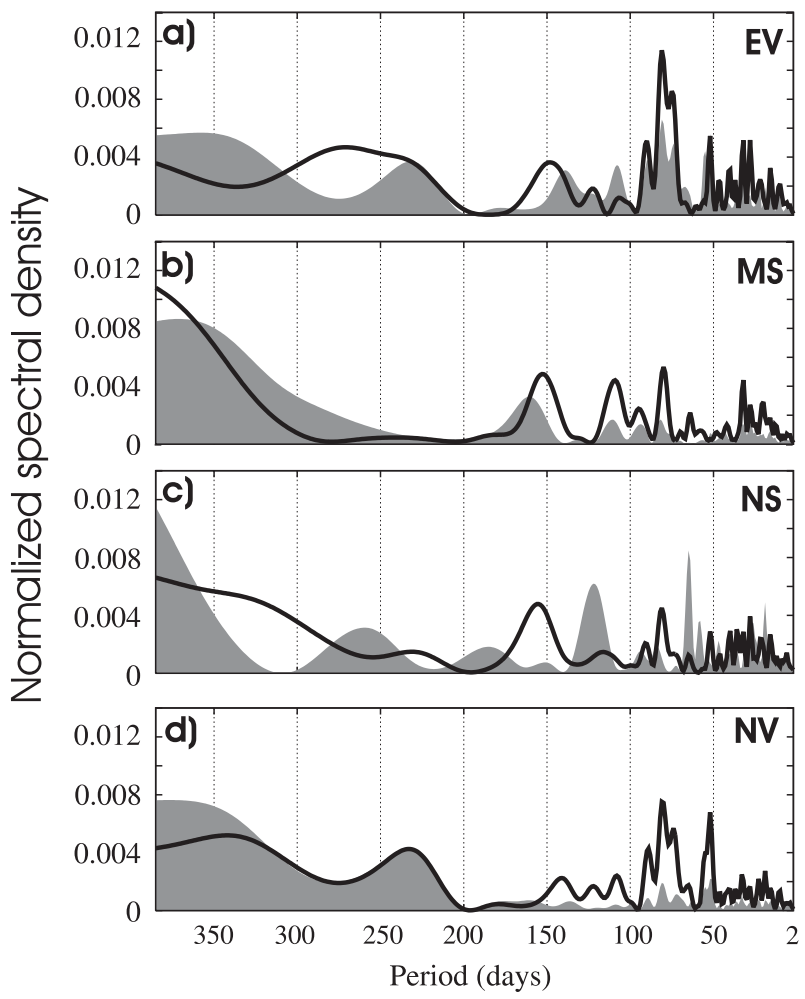

FIG. 11. Spectral analysis of the rotated principal components displayed in Fig. 10. The shaded areas (black lines) represent the normalized spectra calculated with the observational (simulated) series.

analysis is inappropriate and temporal wind variability was assessed with alternative spectral techniques that can be used with unevenly spaced data (Deeming 1975; Belserene 1988; JEA08). Normalized spectra of the simulated and observed rotated principal components in Fig. 11a show that the observed spectrum in the EV subregion is reproduced with some distortion at the lower frequencies. The MS spectra (Fig. 11b) are also quite similar, but with overestimation by the model at the higher frequencies and some displacement at the lower ones with respect to observations. The spectra from the NS subregion do not agree very well (Fig. 11c), which is evidence of poorer wind simulation (see also Figs. 6, 10c). This could be attributed, in part, to the few stations in this subregion and the model's poor representation of the wind variability at two of those sites (15 and 24; Fig. 1). The NV subregion shows qualitative agreement (Fig. 11d) between observational and simulated spectra, with overestimation of the spectral density at the high frequencies but better agreement at lower frequencies. The similarity of spectra for the EV (Fig. 11a) and NV regions (Fig. 11d) highlights their similar wind variability (JEA08). 


\section{SMS}

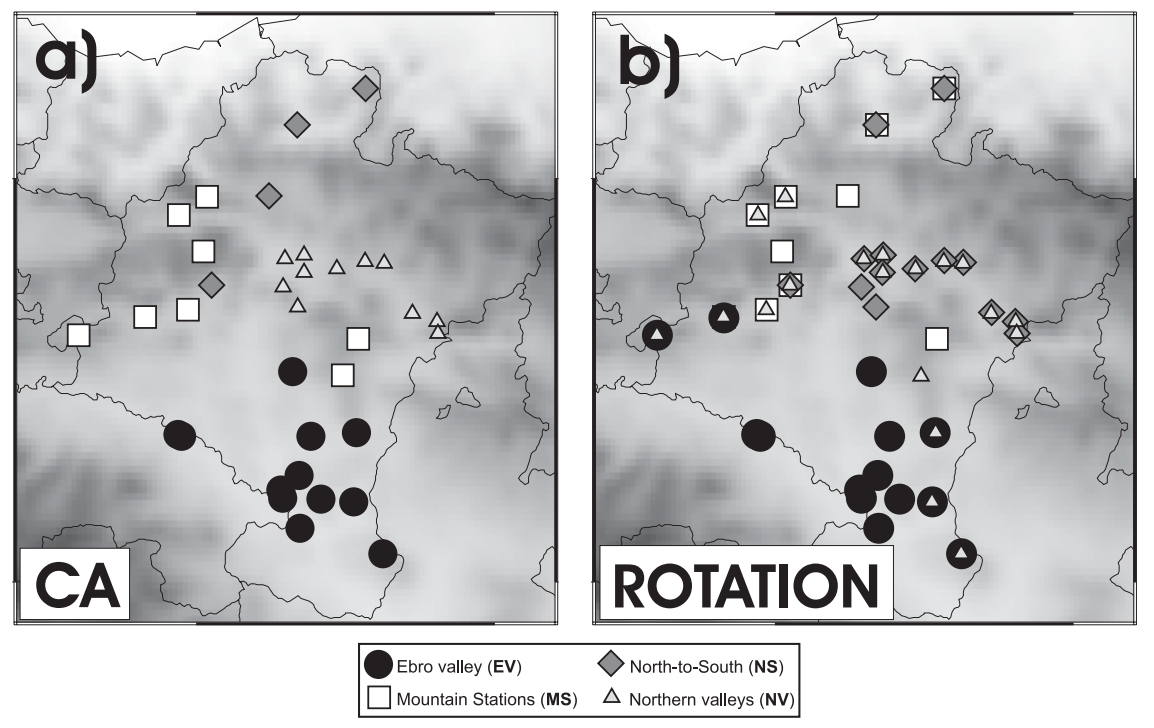

FIG. 12. As in Fig. 9, but for the SMS dataset (Table 1).

\section{c. Inference analysis: The effects of the observational sampling}

The higher spatial and temporal sampling of the WRF simulation is appropriate to further analyze the surface wind variability over the CFN. This analysis leans on the accuracy of the simulation in identifying the wind subregions and reproducing the observed wind variability described in the previous sections.

\section{1) Regionalization OVer the 1992-2005 PERIOD}

The simulation has no missing values, so it can be used to analyze the influence of the observational period on the identification of subregions in JEA08 (1999-2002) and answer the question of how the regionalization is affected when the observational period is extended. The complete time series within the SMS dataset from 1992 to 2005 of the 35 simulated grid points near observational sites is used.

The CA classification (Fig. 12a) compares well with the reduced STMS (Fig. 9a) and observational data (Fig. 3a). The rotational approach (Fig. 12b) also displays similar regions (Fig. 12b) as to those of the reduced STMS classification (Fig. 9b) and those of observations (Fig. 3b). These similarities suggest that the identified wind subregions are robust for the whole observational period from 1992 to 2005.

The rotated SMS principal components permit an analysis of temporal wind variability by subregions for the whole period (1992-2005), without the handicap of missing data. To illustrate this advantage of the simulation over observations, the rotated principal components calculated with the 1999-2002 observational dataset are extended as much as possible and compared against the rotated SMS principal components. With this aim, the standardized anomalies of the observed wind time series for those days with more than 10 wind observations available are projected onto the eigenvector defining each observational subregion; that is, the principal components are evaluated for the 1992-2005 period using the 1999-2002 eigenvectors (see JEA08 for further details). The original rotated principal components and the projections are virtually identical in the overlapping parts (1999-2002). The wavelet power spectra of these projected time series and of the SMS rotated principal components are displayed in Fig. 13. Results for the NS subregion are omitted, because the simulation failed to reproduce the wind variability at this subgroup (Figs. 10c, 11c). Missing observations prevent conclusions from being drawn at the lower frequencies over most of the period (Fig. 13, left), but the power spectra from the simulation do not suffer this limitation (Fig. 13, right). Because the rotated principal component associated with the EV is in concordance with both the zonal and meridional wind component of the subregion (JEA08), the wavelet power spectra of the EV subregion (Fig. 13b) has a structure similar to that calculated with the mean zonal time series (Fig. 8a). The rotated principal component of the MS agrees with the zonal subregion component (JEA08), and its wavelet spectra (Fig. 13d) is similar to that calculated with the 


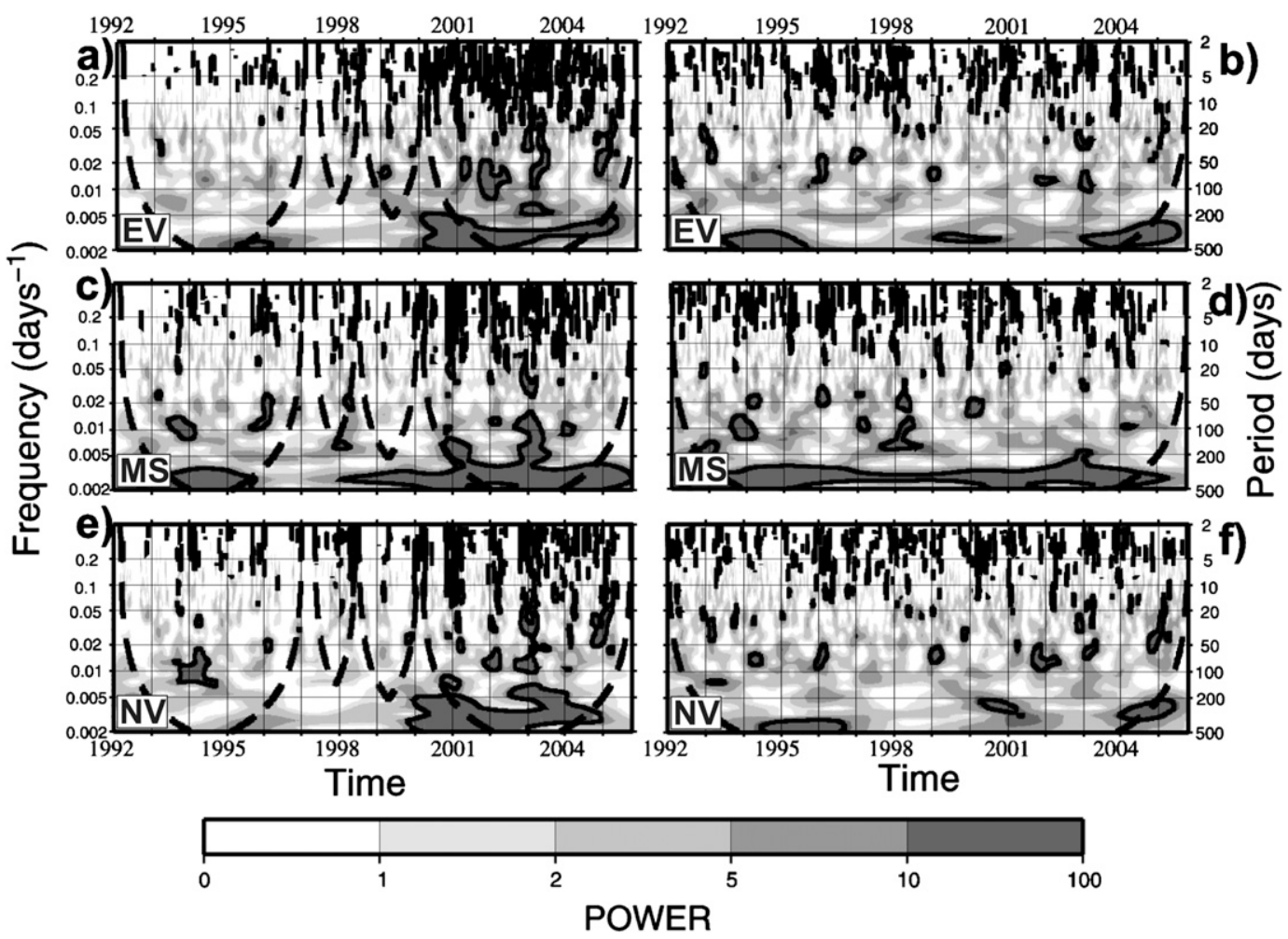

FIG. 13. (left) Wavelet spectral power of the standardized projections calculated with the complete observational dataset and (right) rotated principal components calculated with the SMS dataset for the (top) EV, (middle) MS, and (bottom) NV subregions. As in Fig. 8, but the shaded areas are normalized variances higher than 1, 2, 5, and 10; the black contour lines enclose areas of confidence above $95 \%$ for a first-order autoregressive process, whereas the dashed line represents the cone of influence.

zonal wind of the subregion (Fig. 8b). These similarities support the simulation's ability to reproduce the wind variability in these subregions. The similarities of wind variability in the EV and NV subregions are also captured by the simulation, as indicated by the similarities in wavelet spectra of their principal components (Figs. 13b,f).

\section{2) Regionalization OVer the 1992-2005 PERIOD AND THE FULL DOMAIN}

The limited number of observational sites used in the regionalization (Fig. 1) raises the following question: how might spatial sampling affect the final subgroups? Because the numerical simulation does well in identifying the observed subregions and reproducing their temporal variability, the larger spatial coverage of the simulation can be used to shed some light on this question. With this aim, the wind regionalization is reproduced with the complete simulated dataset and therefore avoiding any temporal or spatial mask.

The wind regions obtained in this exercise with the $\mathrm{CA}$ and the rotational approach lead to the same classification, thus providing robustness to the results achieved.
The visualization of the rotated principal component results is somewhat obscured by the overlapping and the higher density of grid points. The results are shown herein for the CA methodology in Fig. 14. The subregions resemble those obtained with the observed surface wind (Fig. 3a) and the SMS (Fig. 12a). The greater spatial coverage of the simulation allows the identification of other subregions outside the CFN political boundaries. The EV subregion (circles) is well defined by mountain systems that shape the basin. The valleys to the north of the Aralar ridge and the NV subregion are also included in this cluster, indicating a similar temporal variability of these areas. The similar wind variability of the EV and NV was already noticed, but the numerical simulation cannot distinguish them as separate subgroups. The regionalization identifies a subregion (cross symbol) to the east of the CFN that includes some parts of the Ebro valley that had no observations. An MS subregion (squares) includes the Pyrenees from the northern sierras of Bidasoa to Leyre and also some of the mountain sites in the eastern (Izco, Ujue, and San Pedro) and western Sierras (Aralar, Santiago, and Andia; see Fig. 1 for geographical details). However, 


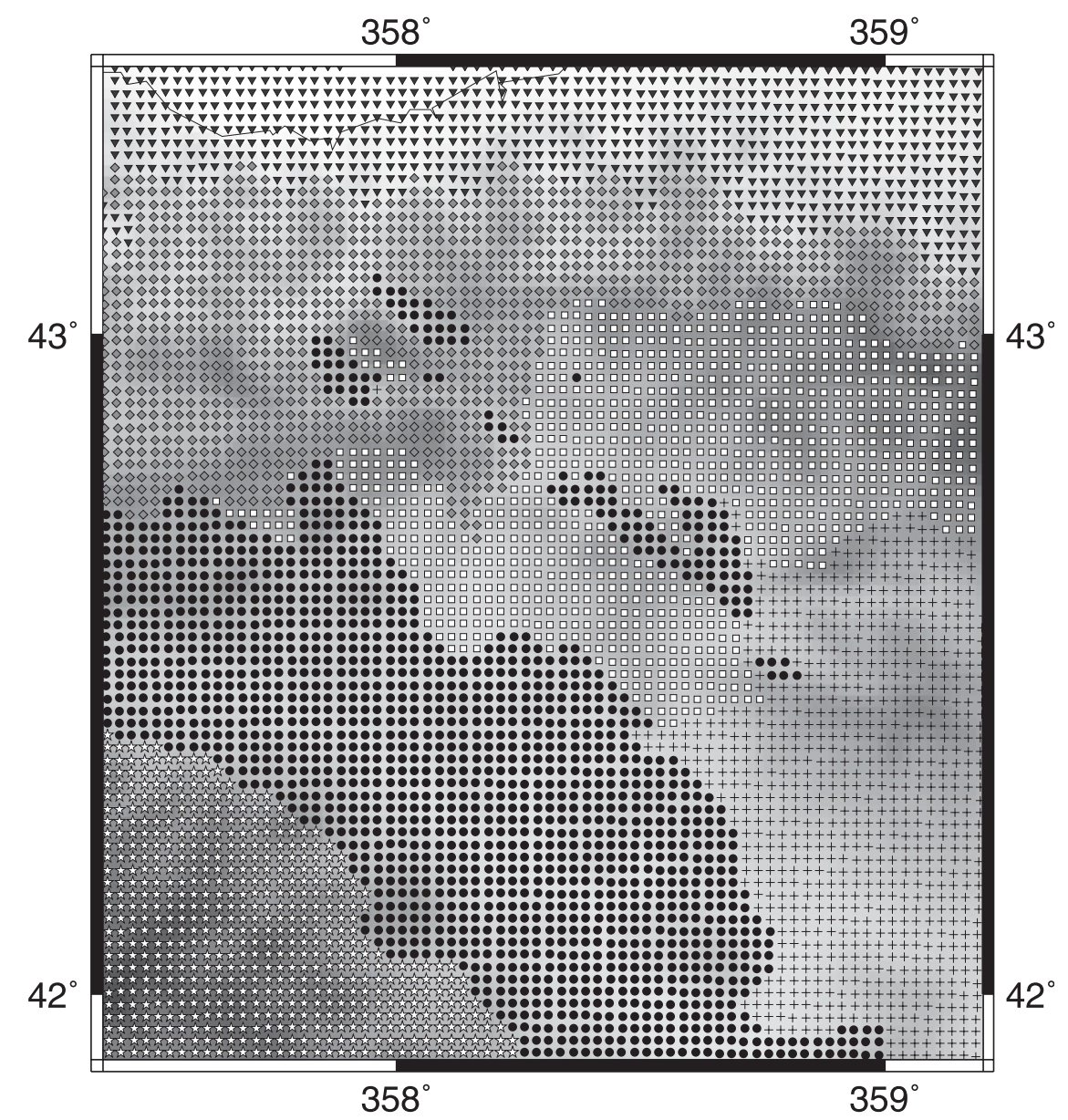

FIG. 14. Regionalization configuration attained after applying the methodology based on the CA to the complete simulated dataset (Table 1). The simulated grid points with similar temporal wind variability are represented with the same symbol and therefore define the identified subregions.

site 20 in the northernmost limits of the CFN is excluded from this group in the simulation, whereas it was an MS site in Fig. 3.

Another mountain subgroup (stars) is found outside the CFN in the Iberian system. The subregion labeled before as NS (diamonds) extends now beyond the previously detected area in the north of the CFN and into surrounding areas. Finally, Fig. 14 shows a subregion (triangles) to the north of the Pyrenees and Cantabrian mountains outside the CFN political boundaries.

\section{Summary and conclusions}

The daily-mean wind variability over complex terrain was simulated at $2-\mathrm{km}$ horizontal resolution with the mesoscale WRF model and compared with observations from the period between 1992 and 2005 for four subregions that enclose observational sites with similar temporal wind variability (EV, MS, NV, and NS), as identified by JEA08. The evaluation at the regional scale rather than at the sites filters out noise associated with local effects or representativeness errors and reinforces the signal; thus, it provides a more appropriate comparison.

Meridional wind variability is more accurately reproduced than zonal wind variability. This is a consequence of the similar meridional wind variability observed over the whole study region, attributed to the influence of large mountain systems adequately represented by the simulation. The zonal variability defines the differences between the wind regions, because it is more affected by the regional features that are not as well represented as the larger-scale topographic features. Wavelet analyses showed reasonably good replication of observed variability by the simulation. It revealed a continuous annual band for the zonal wind component at the mountain subregion and intermittent ones at the valley subregions and for the meridional wind component. 
The model performance was also evaluated by applying the regionalization methodologies to a simulated dataset comparable to observations in its temporal and spatial distribution. The regionalization obtained with the simulation identifies the main characteristics of the subregions, but with some differences in their boundaries. The simulated variability at the EV, MS, and NV subregions is similar to that found in observations. The NS subregion was also detected in the simulation, but its temporal variability was not well reproduced, probably because there were fewer stations in this subregion.

Based on the accuracy shown by the numerical simulation, the WRF results were used to evaluate the effects of the limited observational sampling and the influence of the observational period on the classification of subregions. The latter analysis led to similar subregions, which corroborates the results obtained for the reduced period. The effect of limiting the number of observational sites was investigated by applying the regionalization with the complete simulated wind field over the CFN for the complete simulated period. Virtually the same subregions are identified over the CFN as well as additional subregions outside the observational network coverage, thereby suggesting that even a limited number of sampling stations can reasonably capture the spatial wind variability over the study area.

The results also provide valuable information to improve the observational network. The wind variability at the EV, MS, and NV is appropriately captured, but increasing the number of stations at the NS subregion would be desirable. Some improvement of observational density at mountain tops over the Pyrenees to extend the MS coverage is recommended. In addition, it would be useful if wind observations at the subregions identified outside the network coverage are recorded. Because these subregions are well defined covering large areas, only a few stations in each subregion would be enough to capture their wind variability and provide useful information for future studies.

Acknowledgments. This project was accomplished within the Collaboration Agreement 09/153 between UCM and CIEMAT, and it was partially funded by Project CGL2005-06966-C07/CLI. We thank the Navarra Government for providing us with the wind dataset used in this study and ECMWF for free access to its datasets. An initial version of the wavelet software was provided by C. Torrence and G. Compo (available online at http:// atoc.colorado.edu/research/). We also thank the three reviewers for their helpful comments.

\section{REFERENCES}

Anderson, J. R., E. E. Hardy, J. T. Roach, and R. E. Witmer, 1976: A land use and land cover classification system for use with remote sensor data. U.S. Geological Survey Professional Paper 964, 41 pp.

Baker, R. W., E. W. Hewson, N. G. Butler, and E. J. Warchol, 1978: Wind power potential in Pacific Northwest. J. Appl. Meteor., 17, 1814-1826.

Belserene, E. P., 1988: Rhythms of a variable star. Sky Telesc., 76, 288-290.

Black, T. L., 1994: The new NMC mesoscale Eta Model: Description and forecast examples. Wea. Forecasting, 9, 265-278.

Bliss, N. B., and L. M. Olsen, 1996: Development of a 30-arcsecond digital elevation model of South America. Proc. Pecora 13th Symp. Human Interactions with the EnvironmentPerspectives from Space, Sioux Falls, SD, U.S. Geological Survey.

Buckley, R. L., A. H. Weber, and J. H. Weber, 2004: Statistical comparison of Regional Atmospheric Modelling System forecasts with observations. Meteor. Appl., 11, 67-82.

Cairns, M. M., and J. Corey, 2003: Mesoscale model simulations of high-wind events in the complex terrain of western Nevada. Wea. Forecasting, 18, 249-263.

Conil, S., and A. Hall, 2006: Local regimes of atmospheric variability: A case study of southern California. J. Climate, 19, 4308-4325.

Cotton, W. R., and Coauthors, 2003: RAMS 2001: Current status and future directions. Meteor. Z., 82, 5-29.

Cox, R., B. L. Bauer, and T. Smith, 1998: A mesoscale model intercomparison. Bull. Amer. Meteor. Soc., 79, 265-283.

Deeming, T. J., 1975: Fourier analysis with unequally-spaced data. Astrophys. Space Sci., 36, 137-158.

Dudhia, J., 1989: Numerical study of convection observed during the winter monsoon experiment using a mesoscale twodimensional model. J. Atmos. Sci., 46, 3077-3107.

_ 1996: A multilayer soil temperature model for MM5. Preprints, Sixth PSU/NCAR Mesoscale Model Users' Workshop, Boulder, CO, PSU/NCAR, 49-50.

Endlich, R. M., F. L. Ludwig, C. M. Bhumralkar, and M. A. Estoque, 1982: A diagnostic model for estimating winds at potential sites for wind turbines. J. Appl. Meteor., 21, 1441-1454.

Foufoula-Georgiou, E., and P. Kumar, Eds., 1995: Wavelets in Geophysics. Academic Press, 373 pp.

García, A., J. L. Torres, E. Prieto, and A. de Francisco, 1998: Fitting wind speed distributions: A case study. Sol. Energy, 2, 139-144.

García-Bustamante, E., J. F. González-Rouco, P. A. Jiménez, J. Navarro, and J. P. Montávez, 2008: The influence of the Weibull assumption in monthly wind energy estimation. Wind Energy, 11, 483-502.

,,,,---- and,- 2009 : A comparison of methodologies for monthly wind energy estimations. Wind Energy, 12, $640-659$.

Gesch, D. B., and K. S. Larson, 1996: Techniques for development of global 1-kilometer digital elevation models. Proc. Pecora 13th Symp. Human Interactions with the EnvironmentPerspectives from Space, Sioux Falls, SD, U.S. Geological Survey.

Goodin, W. R., G. J. McRae, and J. H. Seinfeld, 1980: An objective analysis technique for constructing three-dimensional urban scale wind fields. J. Appl. Meteor., 19, 98-108.

Grell, G. A., J. Dudhia, and D. R. Stauffer, 1994: A description of the fifth-generation Penn State/NCAR Mesoscale Model (MM5). NCAR Tech. Note NCAR/TN-398+STR, 122 pp.

Hanna, S. R., and R. Yang, 2001: Evaluations of mesoscale models' simulations of near-surface winds, temperature gradients, and mixing depths. J. Appl. Meteor., 40, 1095-1104. 
Hong, S.-Y., Y. Noh, and J. Dudhia, 2006: A new vertical diffusion package with an explicit treatment of entrainment processes. Mon. Wea. Rev., 134, 2318-2341.

Jackson, P. S., and J. C. R. Hunt, 1975: Turbulent wind flow over a low hill. Quart. J. Roy. Meteor. Soc., 101, 929-955.

Jenkins, G. J., P. J. Mason, W. H. Moores, and R. I. Sykes, 1981: Measurements of the flow structure around Ailsa Craig, a steep, tree-dimensional, isolated hill. Quart. J. Roy. Meteor. Soc., 107, 833-851.

Jiménez, P. A., J. F. González-Rouco, J. P. Montávez, J. Navarro, E. García-Bustamante, and F. Valero, 2008: Surface wind regionalization in complex terrain. J. Appl. Meteor. Climatol., 47, 308-325.

,--1, E. García-Bustamante, and J. Navarro, 2009: Climatology of wind patterns in the northeast of the Iberian Peninsula. Int. J. Climatol., 29, 501-525.

Kain, J. S., and J. M. Fritsch, 1990: A one-dimensional entraining/ detraining plume model and its application in convective parameterization. J. Atmos. Sci., 47, 2784-2802.

- and - 1993: Convective parameterization for mesoscale models: The Kain-Fritsch scheme. The Representation of Cumulus Convection in Numerical Models, Meteor. Monogr., No. 46, Amer. Meteor. Soc., 165-170.

Klink, K., 2002: Trends and interannual variability of wind speed distributions in Minnesota. J. Climate, 15, 3311-3317.

Laprise, R., 1992: The Euler equations of motion with hydrostatic pressure as an independent variable. Mon. Wea. Rev., 120, 197-207.

Lin, Y.-L., R. D. Farley, and H. D. Orville, 1983: Bulk parameterization of the snow field in cloud model. J. Climate Appl. Meteor., 22, 1065-1092.

Lo, J. C.-F., Z.-L. Yang, and R. A. Pielke Sr., 2008: Assessment of three dynamical climate downscaling methods using the Weather Research and Forecasting (WRF) model. J. Geophys. Res., 113, D09112, doi:10.1029/2007JD009216.

Ludwig, F. L., J. M. Livingston, and R. M. Endlich, 1991: Use of mass conservation and critical dividing streamline concepts for efficient objective analysis of winds in complex terrain. J. Appl. Meteor., 30, 1490-1499.

— D. K. Miller, and S. G. Gallaher, 2006: Evaluating a hybrid prognostic-diagnostic model that improves wind forecast resolution in complex coastal topography. J. Appl. Meteor. Climatol., 45, 155-177.

Mahrer, Y., and R. A. Pielke, 1977: The effects of topography on sea and land breezes in a two-dimensional numerical model. Mon. Wea. Rev., 105, 1151-1162.

— M. Segal, and R. Pielke, 1985: Mesoscale modelling of wind energy over non-homogeneous terrain. Bound.-Layer Meteor., 31, 13-23.

Martner, B. E., and J. D. Marwitz, 1982: Wind characteristics in southern Wyoming. J. Appl. Meteor., 21, 1815-1827.

Mason, P. J., and R. I. Sykes, 1979: Flow over an isolated hill of moderate slope. Quart. J. Roy. Meteor. Soc., 105, 383-395.

_ and J. C. King, 1984: Atmospheric flow over a succession of nearly two-dimensional ridges and valleys. Quart. J. Roy. Meteor. Soc., 110, 821-845.

Milligan, G. W., 1980: An examination of the effect of six types of error perturbation on fifteen clustering algorithms. Psychometrika, 50, 325-342.

Mlawer, E. J., S. J. Taubman, P. D. Brown, M. J. Iacono, and S. A. Clough, 1997: Radiative transfer for inhomogeneous atmospheres: RRTM, a validated correlated-k model for the longwave. J. Geophys. Res., 102, 16 663-16 682.
Montgomery, R. B., 1950: The Taylor diagram (temperature against vapour pressure) for air mixtures. Meteor. Atmos. Phys., 2, 163-183.

Pan, Z., E. Takle, W. Gutowski, and R. Turner, 1999: Long simulation of regional climate as a sequence of short segments. Mon. Wea. Rev., 127, 308-321.

Pielke, R. A., 2002: Mesoscale Meteorological Modeling. Academic Press, $676 \mathrm{pp}$.

Preisendorfer, R. W., 1988: Principal Component Analysis in Meteorology and Oceanography. Elsevier, $425 \mathrm{pp}$.

Qian, J.-H., A. Seth, and S. Zebiak, 2003: Reinitialized versus continuous simulations for regional climate downscaling. Mon. Wea. Rev., 131, 2857-2874.

Ratto, C. F., R. Festa, C. Romeo, O. A. Frumento, and M. Galluzzi, 1994: Mass-consistent models for wind fields over complex terrain: The state of the art. Environ. Software, 9, 247-268.

Reid, S., and R. Turner, 2001: Correlation of real and model wind speeds in different terrains. Wea. Forecasting, 16, 620-627.

Reynolds, O., 1895: On the dynamical theory of incompressible viscous fluids and the determination of the criterion. Philos. Trans. Roy. Soc. London, 186B, 123-164.

Rife, D. R., C. A. Davis, Y. Liu, and T. T. Warner, 2004: Predictability of low-level winds by mesoscale meteorological models. Mon. Wea. Rev., 132, 2533-2569.

Sherman, C. A., 1978: A mass-consistent model for wind fields over complex terrain. J. Appl. Meteor., 17, 312-319.

Simmons, A., and J. Gibson, 2000: The ERA-40 Project Plan. ECMWF ERA-40 Project Rep. 1, 63 pp.

Skamarock, W. C., J. B. Klemp, J. Dudhia, D. O. Gill, D. M. Barker, W. Wang, and J. G. Powers, 2005: A description of the advanced research WRF version 2. NCAR Tech. Note TN-468+STR, 88 pp.

Taylor, I. G., 1917: The formation of fog and mist. Quart. J. Roy. Meteor. Soc., 43, 241-288.

Taylor, K. E., 2001: Summarizing multiple aspects of model performance in a single diagram. J. Geophys. Res., 106 (D7), 7183-7192.

Torrence, C., and G. P. Compo, 1998: A practical guide to wavelet analysis. Bull. Amer. Meteor. Soc., 79, 61-78.

Torres, J. L., A. García, E. Prieto, and A. De Francisco, 1999: Characterization of wind speed data according to wind direction. Sol. Energy, 66, 57-64.

,-- M. D. Blas, and A. D. Francisco, 2005: Forecast of hourly average wind speed with ARMA models in Navarre (Spain). Sol. Energy, 79, 65-77.

Troen, I., and E. L. Petersen, 1989: The European Wind Atlas Risoe National Laboratory, $656 \mathrm{pp}$.

Uppala, S. M., and Coauthors, 2005: The ERA-40 Re-Analysis. Quart. J. Roy. Meteor. Soc., 131, 2961-3012.

Verdin, K. L., and S. K. Greenlee, 1996: Development of continental scale digital elevation models and extraction of hydrographic features. Proc. Third Int. Conf./Workshop on Integrating GIS and Environmental Modeling, Santa Fe, NM, National Center for Geographic Information and Analysis.

Volmer, J. P., M. Deque, and D. Rousselet, 1984: EOF analysis of $500 \mathrm{mb}$ geopotential: A comparison between simulation and reality. Tellus, 36A, 336-347.

von Storch, H., 1995: Inconsistencies at the interface of climate impact studies and global climate research. Meteor. Z., 4, 72-80.

_ , and F. W. Zwiers, 1999: Statistical Analysis in Climate Research. Cambridge University Press, 499 pp.

Walmsley, J. L., J. R. Salmon, and P. A. Taylor, 1982: On the application of a model of boundary-layer flow over low hills to real terrain. Bound.-Layer Meteor., 23, 17-46. 
Walter, A., and Coauthors, 2006: A high resolution reference data set of German wind velocity 1951-2001 and comparison with regional climate model results. Meteor. Z., 15, 585-596.

Wendland, W. M., 1982: Wind power as an electrical power source in Illinois. J. Appl. Meteor., 21, 423-428.

Whiteman, C. D., 2000: Mountain Meteorology: Fundamentals and Applications. Oxford University Press, $355 \mathrm{pp}$.
Zagar, N., M. Zagar, J. Cedilnik, G. Gregoric, and J. Rakovec, 2006: Validation of mesoscale low-level winds obtained by dynamical downscaling of ERA40 over complex terrain. Tellus, 58A, 445-455.

Zhong, S., and J. Fast, 2003: An evaluation of the MM5, RAMS, and Meso-Eta models at subkilometer resolution using VTMX field campaign data in the Salt Lake valley. Mon. Wea. Rev., 131, 1301-1322. 